\title{
Human-likeness in utterance generation: effects of variability
}

\author{
Anna Hjalmarsson \& Jens Edlund \\ Centre for Speech Technology, KTH, Stockholm, Sweden \\ \{annah, edlund\}espeech.kth.se
}

\begin{abstract}
There are compelling reasons to endow dialogue systems with human-like conversational abilities, which require modelling of aspects of human behaviour. This paper examines the value of using human behaviour as a target for system behaviour through a study making use of a simulation method. Two versions of system behaviour are compared: a replica of a human speaker's behaviour and a constrained version with less variability. The version based on human behaviour is rated more human-like, polite and intelligent.
\end{abstract}

\section{Introduction}

Content, form and timing all shape our interpretation of utterances as well as their speakers, and how utterances are phrased in spoken dialogue systems (SDSs) affects how the systems are received. [1] states that when designing for people, we should provide a good conceptual model, which allows users to predict the effects of their actions. A plausible model for spoken language is human conversation, in which we by ease express complex meaning; engage in social relationships; and solve problems. However, many human manners (e.g. hesitations; false starts and repetitions; fragmental utterances; and brief feedback) are not in the repertoire of current SDSs, yet research shows that their variation is meaningful, brings information beyond the literal meanings of words, and affect our comprehension [2, 3]. If SDSs are to be more human-like, we need system output that is coherent with human behaviour. This paper introduces a method to test the effects of human behaviour in SDSs: to simulate an SDS behaving very much like a human by replacing one of the parties in a recording of human-human conversation with a synthetic voice. A first study testing the method is presented: non-participating listeners were asked to compare two different versions of SDS behaviour in dialogue.

\section{Data collection and stimuli preparation}

The dialogues used as a basis for this study were collected during the development of the KTH Connector [4], an SDS acting as a personal secretary. Human-human telephony dialogues were collected with 10 subjects: 2 posing as secretaries and 8 as callers. The secretaries took calls over VoIP, and were given a fictional employer's personal agenda. They were asked to answer incoming calls and to act as personal secretaries. The callers were asked to make a phone call to book a meeting. The dialogues are in 
English and each dialogue is about 10 minutes long. All dialogues were transcribed orthographically, including repetitions, hesitations and false starts. Two dialogues were chosen as stimuli for this experiment. In these, the secretary's voice was replaced with a synthetic voice, creating the illusion of a human interacting with an SDS. Two versions were created from each dialogue: a word-by-word replica of the human speaker (UNCONSTRAINED) and a version based on the same set of transcriptions, but constrained lexically through transformations to obtain more limited variability (CONSTRAINED). Similar transformations are suggested in [5]. The following transformations were applied: (a) remove filled pauses, repetitions and false starts; (b) remove any information not directly relevant for the task (banter); (c) reduce lexical variation to one word per meaning only; (d) make brief feedback more explicit - "Ok", for example, was transformed to a full confirmation of the previous utterance such as "ok a Chinese restaurant"; (e) replace pronouns (unless referring to an entity in the same utterance) with the nouns to which they refer; and (f) remove system (secretary) bargeins. The resulting dialogues differ in how information is presented, while preserving the same literal meaning in the context of the conversation: (UnCONSTRAINED) $\mathrm{mm}$ she she has a dinner on Friday mm but she is available on Saturday and Sunday and on Thursday as well; (Constrained) Anna is available for dinner on Thursday Saturday and Sunday. Both versions were produced using Mbrola synthesis [6]. Apart from minor edits to transcriptions, no acoustical adjustments were done. Note that the choice of voice is not principally important in this study: the same voice was used in both cases and the focus of the study was on the lexical content of the utterances rather than on acoustic characteristics.

\section{Method}

23 subjects (15 male and 8 female) between 23 and 65 years of age acted as judges. None had professional experience of speech technology. The test was web-based, with a form and sound clips. Subjects where led to believe the clips were of people interacting with a fully operational SDS. The test contained the two stimuli dialogues, divided into units of about 2-3 utterances each. Each such unit was presented with two sound clips: UnCONSTRAINED and CONSTRAINED, in random order, and subjects were not told how the versions differed. They were asked to compare the two versions on five dimensions, four of which were chosen as they describe characteristics closely associated with human behaviour, the fifth because it is frequently used in SDS evaluations: (1) HuMAN-LIKENESS: the system behaves like a human would do in a similar situation; (2) Politeness: the system acts polite towards the caller; (3) Intelligence: in the context of this dialogue the system behaves intelligently; (4) Display of UNDERSTANDING: the system behaves as if it understands the caller well; and (5) EFFICIENCY: the system tries to help the user in an efficient way. The subjects were further asked to state which version they would prefer, were they to interact with a similar type of system. The subjects chose one of the clips for each dimension, or no difference if they considered the clips equal on some dimension. After completion, they were asked to provide their rating of the importance of the dimensions, on a scale between 1, not important at all, and 5, very important. 


\section{Results}

A McNemar test showed significant differences for Human-Likeness, Politeness and INTELLIGENCE $\quad(p=0.05)$, but no significant differences for EFFICIENCY and UNDERSTANDING, neither was there any preference for a particular version (Figure 1).

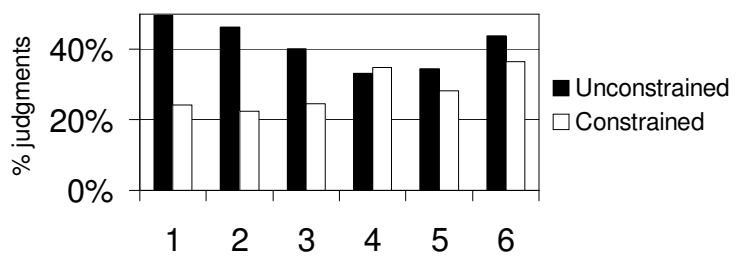

1. HUMAN-LIKENESS

2. POLITENESS

3. INTELLIGENCE

4. UNDERSTANDING

5. EFFICIENCY

6. Preference

Fig. 1 Judgments (\%) distributed over strategies and dimensions

Efficiency in SDSs is traditionally measured quantitatively (e.g. number of words, syllables or utterances to complete a task; see e.g. [7]). To see how such metrics relate to EFFiciency, the qualitative metric, the number of syllables in each system utterance was calculated. The difference in syllables between versions was calculated as a percentage and checked for correlation with EFFICIENCY, but no support for such a correlation was found. EFFICIENCY was also checked for correlations with the other dimensions. A strong correlation (Pearson's correlation coefficient $=0.93$ ) was found between EFFicienCy and UNDERSTANDing (Figure 2). EFFiciency appears to be rated subjectively rather than by the relative length of the dialogues.

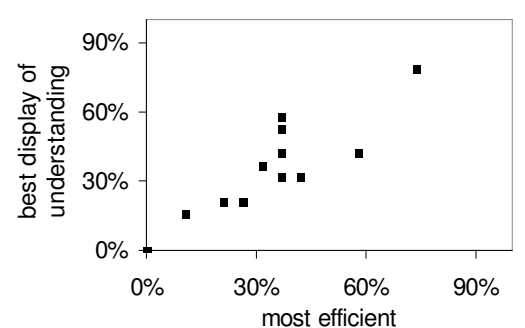

Fig. 2. Correlation between $\%$ EFFICIENCY and \% UNDERSTANDING

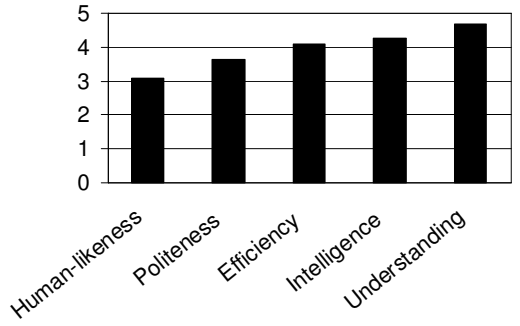

Fig. 3. Mean importance ratings (1-not important at all and 5- very important)

Figure 3 shows the subjects' ratings of the importance of each dimension. All dimensions yield averages $>3$. Politeness and Human-Likeness, both of which were more frequently associated with UNCONSTRAINED, receive the lowest ratings. However, these dimensions were highly correlated with the preferred version (PoLITENess $p=0.93$; HuMAN-LIKENESS $\mathrm{p}=0.72$ ), suggesting that subjects were unaware of what type of behaviour they prefer. This is in line with findings that respondents are often unaware of the processes underlying their decision (see [8] for an overview). 


\section{Discussion}

The results of this study suggest that people have no problems accepting SDSs based on more human behaviour, at least not when judging the interactions of others. The results also support that utterances retaining a larger part of the full spectra of human conversational behaviour are considered more human-like, more intelligent and more polite, compared to utterances with more constrained variability, and that no negative effect on the perception of efficiency and level of understanding can be found. This is interesting since the features removed in the CONSTRAINED version - disfluencies in many accounts - are often regarded as flaws of language. Interestingly, efficiency was not correlated with the utterance length, yet length measures are often used as efficiency measures in SDS evaluations. This may be less of a contradiction than it seems: in task-oriented SDSs, there is often a near-linear relationship between dialogue length and mis- or non-understandings, since there are typically fixed strategies for error handling and utterance generation, and requests for confirmations or repair sequences are only produced when problems occur. Human-human dialogue shows no linear relationship between errors and dialogue length - humans are economic speakers, but not in the same sense as SDSs. Non-task related information, hesitations and repetitions do not necessarily arise as a result of communicative problems.

\section{Acknowledgments}

This research was supported by the Swedish research council project \#2006-2172 (Vad gör tal till samtal/What makes speech special) and project \#2007-6431, GENDIAL. Many thanks to Rolf Carlson and Joakim Gustafson.

\section{References}

1. Norman, D. A.: The design of everyday things, MIT Press (1988)

2. Brennan, S. E.: Processes that shape conversation and their implications for computational linguistics. In Proceedings of the $38^{\text {th }}$ Annual Meeting of the ACL, Hong Kong, (2000)

3. Arnold, J. E., Fagano, M., and Tanenhaus, M. K.: Disfluencies signal theee, um, new information. Journal of Psycholinguistic Research, Vol. 32 (2003) 25-36

4. Edlund, J. and Hjalmarsson, A.: Applications of Distributed Dialogue Systems: the KTH Connector. In Proceedings of ISCA Tutorial and Research Workshop on Applied Spoken Language Interaction in Distributed Environments (ASIDE 2005) Aalborg (2005)

5. Jönsson, A. and Dahlbäck, N.: Distilling dialogues - a method using natural dialogue corpora for dialogue systems development. In Proceedings of the 6th Applied Natural Language Processing Conference, Seattle (2000)

6. Dutoit, T., Pagel, V., Pierret, N., Bataille, F., \& Vreken, O. v. d.: The MBROLA project: Towards a set of high-quality speech synthesizers free of use for non-commercial purposes. In Proceedings of ICSLIP ' 96 , (1996) 1393-1396.

7. Walker, M., Kamm, C., and Litman, D.: Towards developing general models of usability with PARADISE. Natural Language Engineering: Special Issue on Best Practice in Spoken Dialogue Systems, September (2000)

8. Nisbett, R. E. and Wilson, T. D.: Telling More Than We Know: Verbal Reports on Mental Processes. Psychological Review, Vol. 84 (3) (1977) 231-59 